\title{
A importância da atuação do profissional de enfermagem na assistência preventiva à Depressão Puerperal: uma revisão integrativa
}

\author{
The importance of the performance of nursing professionals in preventive care for \\ Puerperal Depression: an integrative review
}

La importancia del desempeño del profesional de enfermería en la asistencia preventiva a la Depresión Puerperal: una revisión integradora

Cleice Luane Silveira de Carvalho Brandão ${ }^{1}$, Erirayne Simplicio Quadro do Nascimento ${ }^{1 *}$, Camila Monique Souza de Oliveira Aramaio'.

\begin{abstract}
RESUMO
Objetivo: Analisar através das produções científicas, a importância da atuação do profissional de enfermagem na assistência preventiva ao surgimento da depressão puerperal. Métodos: Utilizou-se como metodologia de pesquisa um estudo bibliográfico do tipo revisão integrativa com abordagem qualitativa no período de 2016 a 2021. Os dados foram coletados utilizando-se Descritores em Ciências da Saúde (DeCS/MeSH) mediante as bases de dados da Biblioteca Virtual em Saúde (BVS), Google Acadêmico e Scientific Electronic Library Online (SCIELO). Utilizou-se como descritores: "Assistência de Enfermagem", "Depressão Puerperal", "Prevenção e Atenuação". Resultados: Foram encontrados 1805 artigos nas bases de dados. Após a aplicação dos critérios de exclusão, foram selecionadas 12 pesquisas para serem analisadas subsidiando a revisão integrativa. Os principais resultados na pesquisa realizada demonstram que não existe uma infraestrutura adequada para a atuação do profissional de enfermagem no Brasil para acolher de forma preventiva a paciente mediante a identificação e o agravamento da Depressão Puerperal, sendo que é imprescindível a sua atuação para o efetivo tratamento. Considerações finais: Observou-se a importância do profissional de Enfermagem para a prevenção à DPP, fazendo-se necessário a capacitação profissional e estruturação das clínicas e hospitais, a fim de conferir condições mínimas necessárias para a atuação preventiva.
\end{abstract}

Palavras-chave: Assistência de enfermagem, Depressão puerperal, Prevenção e atenuação.

\begin{abstract}
Objective: To analyze through scientific productions, the importance of the performance of nursing professionals in preventive care to the onset of puerperal depression. Methods: A bibliographic study of the integrative review type with a qualitative approach from 2016 to 2021 was used as the research methodology. The data were collected using Health Sciences Descriptors (DeCS/MeSH) through the databases of the Library Virtual Health (VHL), Google Scholar and Scientific Electronic Library Online (SCIELO). The following descriptors were used: "Nursing Assistance", "Puerperal Depression", "Prevention and Attenuation". Results: 1805 articles were found in the databases. After applying the exclusion criteria, 12 surveys were selected to be analyzed supporting the integrative review. The main results in the research carried out demonstrate that there is not an adequate infrastructure for the performance of the nursing professional in Brazil to preventively welcome the patient through the identification and aggravation of Puerperal Depression, and its performance is essential for effective treatment. Final considerations: It was observed the importance of the nursing professional for the prevention of PPD, making it necessary the professional training and structuring of clinics and hospitals, in order to provide the minimum conditions necessary for preventive action.
\end{abstract}

Key words: Nursing assistance, Puerperal depression, Prevention and attenuation.

\footnotetext{
${ }^{1}$ Centro Universitário Aparício Carvalho (FIMCA), Porto Velho - RO. * E-mail: erirraynesimplicio2020@gmail.com
}

PUBLICADO EM: 5/2021 


\section{RESUMEN}

Objetivo: Analizar a través de producciones científicas, la importancia del desempeño de los profesionales de enfermería en la atención preventiva ante la aparición de la depresión puerperal. Métodos: Se utilizó como metodología de investigación un estudio bibliográfico de tipo revisión integradora con enfoque cualitativo de 2016 a 2021. Los datos fueron recolectados utilizando Descriptores de Ciencias de la Salud (DeCS/MeSH) a través de las bases de datos de la Biblioteca Virtual de Salud (BVS), Google Académico e Scientific Electronic Library Online (SCIELO). Se utilizaron los siguientes descriptores: "Asistencia de enfermería", "Depresión puerperal", "Prevención y atenuación". Resultados: Se encontraron 1805 artículos en las bases de datos. Luego de aplicar los criterios de exclusión, seleccionaron 12 encuestas para ser analizadas apoyando la revisión integradora. Los principales resultados de la investigación realizada demuestran que no existe una infraestructura adecuada para desempeño del profesional de enfermería en Brasil para acoger preventivamente al paciente mediante la identificación y agravamiento de la Depresión Puerperal, desempeño es fundamental para tratamiento efectivo. Consideraciones finales: Se observó la importancia del profesional para la prevención de la DPP, haciendo necesaria la formación y estructuración profesional de clínicas y hospitales, a fin de brindar las condiciones mínimas necesarias para acción preventiva.

Palabras clave: Asistencia de enfermería, Depresión puerperal, Prevención y atenuación.

\section{INTRODUÇÃO}

Durante o período gestacional, o comportamento feminino sofre algumas alterações em razão de mudanças hormonais, apresentando humor mais instável, bem como sentimentos conflitantes em relação a sua vida e sua nova realidade (ALMEIDA NMC e ARRAIS AR, 2016). A partir do momento em que a mulher adquire a consciência de seu novo papel na família e na sociedade, as questões mais importantes relacionadas a sua vida também sofrem profundas transformações. No puerpério, que corresponde ao período imediato ao nascimento da criança, é marcado pela queda hormonal e fatores emocionais que podem causar um estado letárgico na mulher durante algum tempo, sendo inerente ao processo gravídico-puerperal (AYOAMA EA, et al., 2019). Dessa forma, o estado puerperal é natural e sua duração é de curto prazo, não constituindo uma patologia psíquica ou física prejudicial à parturiente.

A Depressão Puerperal ou Depressão Pós-Parto (DPP) é uma doença classificada como um transtorno psíquico em decorrência do estado puerperal identificada pela CID-11 (SILVA CRA, et al., 2020). Assim, devido à alteração de sua identidade enquanto mulher para o desempenho de sua função materna, vários fatores influenciam o surgimento dessa patologia. Ainda, conforme os autores supracitados, na fase puerperal é natural a presença de mudanças de natureza emocional em razão do impacto psicológico, social, econômico e familiar que o nascimento de uma criança proporciona. Contudo, a patologia psíquica DPP vai além do estado reflexivo natural, influenciando nos cuidados da mãe em relação ao seu bebê e sua condição psíquica enquanto durar o puerpério (SILVA CRA, et al., 2020).

Nesse sentido, o acompanhamento pelos profissionais de enfermagem exerce a função fundamental de observação especializada ainda no período gestacional a fim de constatar indícios de uma possível DPP (AYOAMA EA, et al., 2019). Ocorre que a depressão puerperal é uma patologia psíquica complexa, de difícil constatação precoce. Pois, a sua gênese envolve fatores de riscos de diversas naturezas: hormonais, emocionais, psicológicas, econômicas, sociais etc. Consequentemente, conforme os autores supracitados, na fase pré-natal é possível a verificação de determinados comportamentos que envolvem a vida da gestante, os quais possuem potencial "depressor" das emoções (AYOAMA EA, et al., 2019).

Os fatores de riscos envolvendo o puerpério são inerentes à condição do ciclo gravídico-puerperal, no qual busca-se contrabalancear por meio de fatores de proteção (ALMEIDA NMC e ARRAIS AR, 2016). Nesse contexto, $O$ atendimento individual e pessoal realizado pelos profissionais de enfermagem influenciam diretamente no processo de prevenção à DPP (GOMES BKA, et al., 2019). Assim, há uma relação entre a equipe de enfermagem especializada e a recepção da parturiente a essa nova fase de sua vida, compreendendo a humanização no atendimento conforme as suas particularidades. 
O atendimento humanizado, portanto, realizado pelos profissionais de enfermagem cumprem a sua função precípua de atender às necessidades do ser humano enquanto sob sua supervisão, assistindo-o e buscando o seu bem-estar (GONÇALVES FBAC e ALMEIDA MC, 2019). Conforme os autores supracitados, é importante a qualidade da assistência oferecida pela equipe de enfermagem à mulher em seu ciclo gravídicopuerperal, bem como orientações acerca do seu estado físico e psicológico a fim de evitar o surgimento ou agravamento da DPP. Trata-se, dessa maneira, de uma atuação preventiva, sobretudo. Diante disso, é essencial esse enfretamento, pois os efeitos do agravamento dessa patologia psicológica afetam não apenas a mãe, como também a criança e sua família (VIANA MDZS, et al., 2020).

A DPP acomete cerca de $25 \%$ das mulheres no Brasil (FIOCRUZ, 2016) representado uma doença relevante para a saúde pública da sociedade brasileira. Apesar da sua incidência na mulher, os seus efeitos refletem diretamente nas pessoas que com ela convive, especialmente o recém-nascido e a família (TOLENTINO E, et al., 2016). Portanto, o profissional de enfermagem realiza uma série de atos que resultam no contado direito com a gestante e puérpera (GUIMARÃES RB, et al., 2021) durante seu ciclo gravídicopuerperal. Por conseguinte, busca-se a união entre o sistema de saúde e a mulher a fim de enfrentar essa fase mediante as medidas preventivas e superar a DPP (RIBEIRO NM, et al., 2019).

O presente estudo objetiva, analisar por meio das produções científicas a importância da atuação do profissional de enfermagem na assistência preventiva ao surgimento da depressão puerperal. Contudo, é necessário compreender os elementos presentes nessa relação ente a mãe, o recém-nascido, sua família e a equipe de enfermagem. Diante na fragilidade emocional no estado puerperal e suas consequências potencialmente prejudiciais, a temática proposta é de grande relevância para a saúde pública, meio acadêmico e sociedade. Assim, a fim de responder ao objetivo da presente pesquisa científica, apresenta-se como pergunta norteadora: "Qual a importância da atuação do profissional de enfermagem na assistência preventiva à depressão puerperal através das produções científicas?”.

\section{MÉTODOS}

Utilizou-se como metodologia de pesquisa um estudo bibliográfico do tipo revisão integrativa com abordagem qualitativa no período de 2016 a 2021. Os dados foram coletados utilizando-se Descritores em Ciências da Saúde (DeCS/MeSH) mediante as bases de dados da Biblioteca Virtual em Saúde (BVS), Google Acadêmico e Scientific Electronic Library Online (SCIELO).

A revisão integrativa proporciona a fundamentação da temática, auxiliando na composição de obras científicas, sua análise conclusiva e progressiva (MENDES KDS, et al., 2008). As etapas da revisão integrativa consistem é um método de pesquisa não-experimental, pelo qual se realiza a análise de produções científicas mediante alguns critérios pré-definidos pelo autor, passando por etapas de pesquisa, seleção, exclusão, interpretação e síntese dos dados (SOUZA MT, et al., 2010). Reúnem-se e sintetizam-se estudos que tenham relevância com o assunto abordado e, por meio dos resultados evidenciados, obtém-se uma conclusão.

Estabeleceram-se como critérios de inclusão artigos originais, disponíveis na íntegra e com livre acesso aos interessados, em língua portuguesa publicados no período entre 2016 a 2021. Excluíram-se os artigos científicos duplicadas, monografias, dissertações, teses, comentários autorais, editoriais e cartas. Portanto, obras que não se enquadraram nos parâmetros da presente pesquisa foram exclusas. Utilizaram-se, para a busca, os DeCS: Assistência de Enfermagem, Depressão Puerperal, Prevenção e Atenuação.

Mediante a pesquisa realizada nas bases de dados, foram encontrados 1805 artigos científicos dentro dos parâmetros elencados. Primeiramente, foram excluídos 23 estudos por se apresentarem como indisponíveis para a consulta; posteriormente, 756 foram exclusos por apresentar período de publicação fora dos parâmetros desse estudo; por fim, 1006 artigos não atendiam à temática proposta pela presente pesquisa, sendo igualmente excluídos do estudo. Portanto, após a análise realizada foram selecionados 20 artigos científicos para a leitura na íntegra e sua análise para a composição desse estudo científico, dos quais foram excluídos 8 artigos em razão de utilizar revisão integrativa. Por fim, foram selecionados 12 artigos com o escopo de subsidiar a revisão de literatura (Figura 1). 
Figura 1 - Fluxograma das etapas para a seleção dos artigos desta revisão integrativa, 2021.

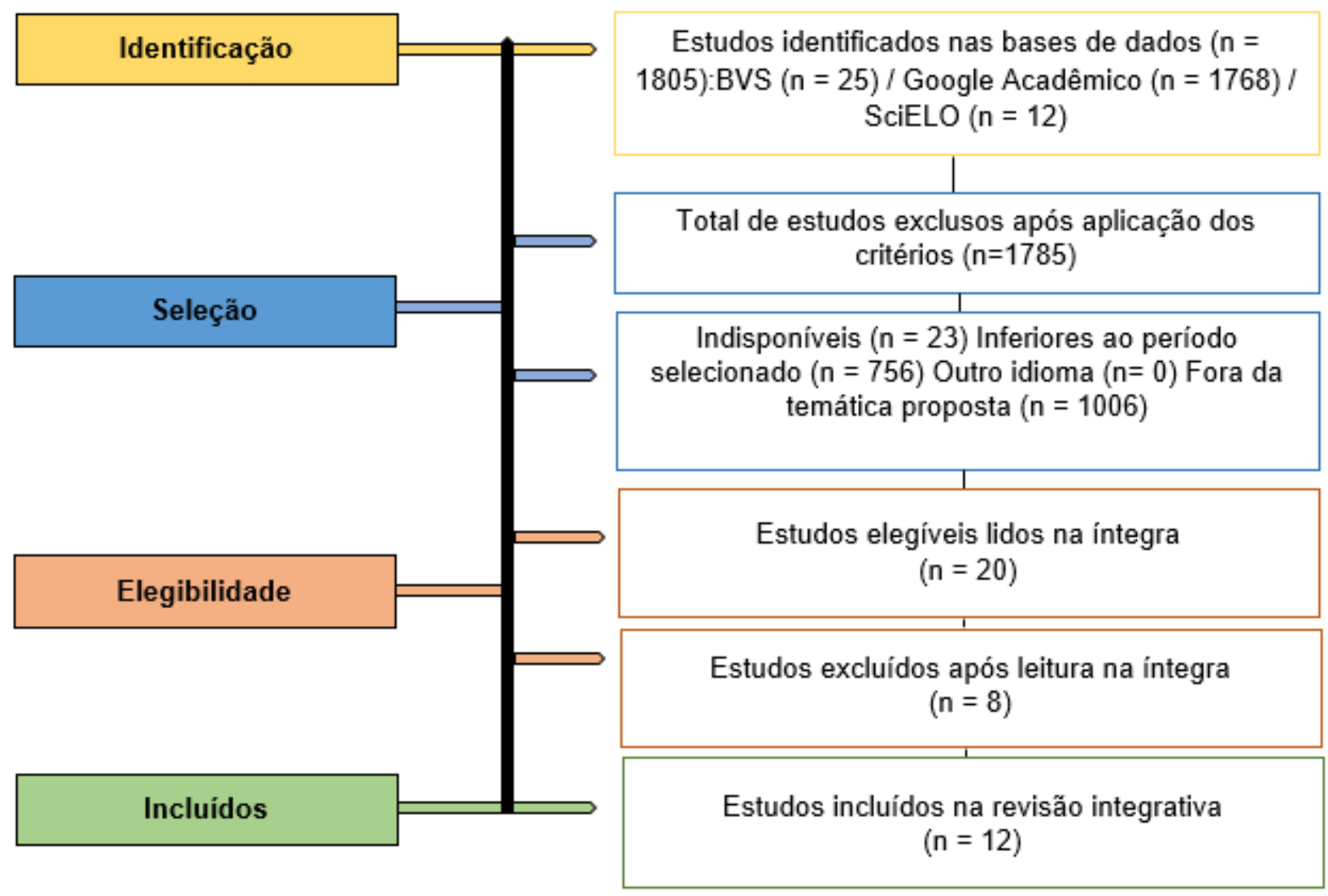

Fonte: Brandão CLSC, et al., 2021.

\section{RESULTADOS E DISCUSSÃO}

Selecionaram-se 12 artigos científicos que respondiam à questão de pesquisa. Deste modo, elaborou-se uma síntese analítica dos resultados apresentados elencados em uma tabela específica, na qual contém: a) os autores; b) ano; c) local; d) periódico de publicação; e) tipo de pesquisa; f) as vertentes das pesquisas quanto à importância da atuação do profissional de enfermagem na assistência preventiva à depressão puerperal.

No que se refere ao tipo de estudo, verificou-se que a maioria adotou a abordagem descritiva (Quadro 1). Tratam-se, portanto, de obras científicas de cunho analítico em sua maioria, cujo objetivo principal é verificar a relevância da atuação do profissional de enfermagem no enfrentamento à DPP. O conhecimento científico é construído de forma contínua e colaborativa por meio de pesquisadores e cientistas. 
Quadro 1 - Características dos artigos inclusos neste estudo, de acordo com os autores, ano, localidade e periódico de publicação, tipo de estudo e vertentes compreendendo a atuação dos profissionais de enfermagem no enfrentamento preventivo ao DPP dos estudos analisados, 2021.

\begin{tabular}{|c|c|c|c|}
\hline Autores & $\begin{array}{l}\text { Ano, localidade e } \\
\text { periódico de } \\
\text { publicação }\end{array}$ & $\begin{array}{l}\text { Tipo de } \\
\text { estudo }\end{array}$ & Vertente do artigo \\
\hline $\begin{array}{l}\text { SANTOS FK, } \\
\text { et al. }\end{array}$ & $\begin{array}{l}\text { 2020/ Minas Gerais } \\
\text { (MG)/ Revista Nursing }\end{array}$ & $\begin{array}{l}\text { Qualitativo e } \\
\text { Descritivo }\end{array}$ & $\begin{array}{l}\text { Analisar a percepção de enfermeiros } \\
\text { sobre o diagnóstico, acompanhamento, } \\
\text { tratamento e alta de mulheres portadoras } \\
\text { de DPP no município de Divinópolis-MG. }\end{array}$ \\
\hline $\begin{array}{l}\text { ALOISE SR, } \\
\quad \text { et al. }\end{array}$ & $\begin{array}{l}\text { 2019/ Manaus (AM)/ } \\
\text { Enfermagem em Foco }\end{array}$ & $\begin{array}{l}\text { Quantitativo } \\
\text { e Descritivo } \\
\text { Transversal }\end{array}$ & $\begin{array}{c}\text { Identificar sinais e sintomas de DPP e } \\
\text { determinar o perfil socioeconômico e } \\
\text { obstétrico das participantes a fim de } \\
\text { apontar possíveis fatores associados à } \\
\text { doença. }\end{array}$ \\
\hline $\begin{array}{l}\text { JORDÃO } \\
\text { RRR, et al. }\end{array}$ & $\begin{array}{l}\text { 2017/ Recife (PE)/ Rev. } \\
\text { Eletr. Enf. [Internet]. }\end{array}$ & $\begin{array}{l}\text { Quantitativo e } \\
\text { Descritivo }\end{array}$ & $\begin{array}{c}\text { Investigar a acurácia de um conjunto de } \\
\text { CDs do diagnóstico Desempenho do papel } \\
\text { ineficaz no contexto de puérperas } \\
\text { atendidas em Unidades de Saúde da } \\
\text { Família (USF) }\end{array}$ \\
\hline $\begin{array}{l}\text { SOUZA KLC, } \\
\text { et al. }\end{array}$ & $\begin{array}{l}\text { 2018/ Recife (PE)/ Rev } \\
\text { enferm UFPE online. }\end{array}$ & $\begin{array}{l}\text { Qualitativo e } \\
\text { Descritivo }\end{array}$ & $\begin{array}{l}\text { Analisar o conhecimento dos enfermeiros } \\
\text { das unidades de saúde da família sobre a } \\
\text { depressão puerperal. }\end{array}$ \\
\hline $\begin{array}{l}\text { MOLL MF, et } \\
\quad \text { al. }\end{array}$ & $\begin{array}{l}\text { 2019/ Recife (PE)/ Rev } \\
\text { enferm UFPE online. }\end{array}$ & $\begin{array}{l}\text { Quantitativo, } \\
\text { Descritivo, } \\
\text { Exploratório, } \\
\text { Transversal }\end{array}$ & $\begin{array}{l}\text { Rastrear a depressão pós-parto entre } \\
\text { mulheres jovens que estão na segunda } \\
\text { semana e no sexto mês após o parto. }\end{array}$ \\
\hline $\begin{array}{l}\text { OLIVEIRA } \\
\text { AM, et al. }\end{array}$ & $\begin{array}{l}\text { 2016/ Pelotas (RS)/ J } \\
\text { Nurs Health. }\end{array}$ & $\begin{array}{l}\text { Qualitativo e } \\
\text { Descritivo }\end{array}$ & $\begin{array}{l}\text { Investigar o conhecimento de profissionais } \\
\text { da Estratégia Saúde da Família quanto ao } \\
\text { tratamento da depressão pós-parto (DPP) }\end{array}$ \\
\hline $\begin{array}{l}\text { BOSKA GA, et } \\
\text { al. }\end{array}$ & $\begin{array}{l}\text { 2016/ Pelotas (RS)/ J } \\
\text { Nurs Health. }\end{array}$ & $\begin{array}{l}\text { Transversal, } \\
\text { Descritivo }\end{array}$ & $\begin{array}{c}\text { Identificar sintomas depressivos e } \\
\text { associá-los às características } \\
\text { sociodemográficas e clínicas de mulheres } \\
\text { no puerpério tardio. }\end{array}$ \\
\hline $\begin{array}{l}\text { AYOAMA EA, } \\
\text { et al. }\end{array}$ & $\begin{array}{c}\text { 2019/ Curitiba (PR)/ } \\
\text { Braz. J. Hea. Rev. }\end{array}$ & $\begin{array}{l}\text { Descritivo e } \\
\text { Observatório }\end{array}$ & $\begin{array}{c}\text { Conscientizar o profissional de } \\
\text { enfermagem a importância de uma } \\
\text { consulta de pré-natal qualificada para } \\
\text { identificação das gestantes com sinais e } \\
\text { sintomas de depressão gestacional. }\end{array}$ \\
\hline $\begin{array}{l}\text { ALMEIDA } \\
\text { NMC e } \\
\text { ARRAIS AR }\end{array}$ & $\begin{array}{l}\text { 2016/ Brasília (DF)/ } \\
\text { Psicol.: ciênc. prof. }\end{array}$ & $\begin{array}{l}\text { Descritivo e } \\
\text { Pesquisa- } \\
\text { Ação }\end{array}$ & $\begin{array}{c}\text { Avaliar a eficácia do PNP na prevenção à } \\
\text { DPP em gestantes de alto risco internadas } \\
\text { em um hospital público de referência, em } \\
\text { Brasília. }\end{array}$ \\
\hline $\begin{array}{l}\text { GONÇALVES } \\
\text { FBAC e } \\
\text { ALMEIDA MC }\end{array}$ & $\begin{array}{l}\text { 2019/ Brasília (DF)/ } \\
\text { Ensaios e Ciênc. }\end{array}$ & $\begin{array}{l}\text { Descritivo e } \\
\text { Narrativo. }\end{array}$ & $\begin{array}{l}\text { Relata a DPP e aponta os aspectos de } \\
\text { riscos que podem colaborar para esta } \\
\text { síndrome, sua primazia e as escalas mais } \\
\text { utilizadas pelos profissionais da saúde. }\end{array}$ \\
\hline $\begin{array}{l}\text { TOLENTINO } \\
\text { E, et al. }\end{array}$ & $\begin{array}{l}\text { 2016/ João Pessoa (PB). } \\
\text { Rev. Ciênc. Saúde Nova } \\
\text { Esperança. }\end{array}$ & Qualitativo & $\begin{array}{l}\text { Discutir sobre os sinais e sintomas da } \\
\text { depressão pós-parto em puérperas e } \\
\text { permitir a visibilidade deste assunto para } \\
\text { as mães puérperas, profissionais da área, } \\
\text { bem como para a sociedade em geral. }\end{array}$ \\
\hline $\begin{array}{l}\text { RIBEIRO NM, } \\
\text { et al. }\end{array}$ & $\begin{array}{l}\text { 2019/ Bom Jesus do } \\
\text { Itabapoana (RJ) Revista } \\
\text { Científica } \\
\text { Interdisciplinar }\end{array}$ & Descritivo & $\begin{array}{l}\text { Conhecer mais sobre a Depressão Pós- } \\
\text { Parto, mostrando a importância do } \\
\text { cuidado de enfermagem a gestante no } \\
\text { pré-natal e puerpério e compreender as } \\
\text { ações da equipe de enfermagem que } \\
\text { atuam dentro do pré-natal. }\end{array}$ \\
\hline
\end{tabular}

Fonte: Brandão CLSC, et al., 2021. 
Conforme análise das características do quadro supra elaborado, 92\% dos artigos científicos realizaram a pesquisa mediante a abordagem descritiva. Os quais buscam, cada qual conforme a sua vertente, compreender as necessidades da mulher em seu ciclo gravídico-puerperal no que se refere à DPP. Assim, constata-se a importância da atuação do profissional de enfermagem no tratamento preventivo à depressão puerperal.

Em relação à DPP, as atuações dos profissionais de enfermagem devem direcionar seus conhecimentos para uma diversidade de demandas com as quais mantém contato direto diuturnamente (RIBEIRO NM, et al., 2019). Problemas de natureza psicológica são constantes na sociedade, sendo, contudo, de difícil constatação. Dessa forma, exige-se dos enfermeiros maior sensibilidade para identificar de forma precoce alguma predisposição depressiva, bem como instruir e encaminhar mulheres gestantes e puérperas conforme a gravidade do caso.

Dessa forma, a assistência à mulher na prevenção à depressão puerperal é uma atividade que exige do profissional de enfermagem uma atuação sensível e particularizada, buscando verificar as características específicas da parturiente. Não há uma resposta definitiva a essa problemática, cabendo a atuação dos pesquisadores a sua investigação, observação e descrição. Assim, as análises proporcionam uma maior consciência desse profissional no desempenho de seu labor humanitário. Nesse sentido, os autores são uníssonos em suas vertentes no que se refere ao conhecimento dessa modalidade de depressão para a consecução de seus objetivos.

Mediante a análise dos artigos científicos selecionados, observou-se que $100 \%$ deles são unânimes quanto a importância do profissional de enfermagem para a assistência preventiva à depressão puerperal. Contudo, verificou-se que $8,33 \%$ das pesquisas analisadas apresentou não haver relação entre as variáveis sociais, econômicas e clínico-obstétricas com a presença da DPP (ALOISE SR, et al., 2019). Portanto, a maioria dos estudos realizados apontam para a relação entre essas variáveis como fatores de riscos para o surgimento ou agravamento da depressão na puérpera. Sendo, dessa forma, necessária a avaliação pelos enfermeiros (as) das respectivas condições e situações cotidianas (JORDÃO RRR, et al, 2017).

Ainda, $41,66 \%$ das pesquisas concordam no que se refere à falta de capacitação especializada desses profissionais para atender à mulher em seu ciclo gravídico-puerperal com fins de prevenção à DPP (OLIVEIRA $\mathrm{AM}$, et al., 2016). É necessário, portanto, uma abordagem não meramente física e biológica, mas também psicológica (ALMEIDA NMC e ARRAIS AR, 2016). No mesmo sentido, observou-se que $25 \%$ dos artigos apresentam como elemento prejudicial a ausência de estrutura dos hospitais e clínicas para o acolhimento efetivo da parturiente no que se refere ao tratamento preventivo da depressão puerperal (SANTOS FK, et al., 2020).

Mediante a análise das produções científicas, verificou-se que existe uma necessidade de maior atenção às gestantes e puérperas em seu cotidiano, buscando identificar sintomas relacionados à DPP (JORDÃO RRR, et al., 2017). Essa atividade dos profissionais de enfermagem é essencial, pois os vestígios dessa patologia não se verificam de forma concreta e objetiva, mas abstrata, exigindo dos enfermeiros habilidades de observação comportamental dessas mulheres. Ocorre que o cerne do tratamento realizado em clínicas e hospitais é majoritariamente direcionado aos cuidados com a criança, bem como a necessidades fisiológicas e reprodutivas (SOUZA KLC, et al., 2018). Desse modo, a formação dos profissionais de enfermagem em relação ao acolhimento realizado à mulher em seu período gravídico-puerperal é superficial no enfrentamento preventivo da depressão puerperal.

Ainda, os fatores de risco ao surgimento e agravamento da DPP possuem relação direta com aspectos assistenciais e sociais (MOLL MF, et al., 2019). Nesse sentido, é necessário a construção de um plano de atendimento integral à mulher, considerando-se os referidos dados sociodemográficos e suas características individuais. Conforme analisando nas pesquisas, a depressão puerperal possui inúmeros fatores de risco relacionados às diversas searas de sua vivência. Assim, a assistência a essas mulheres implica em uma atuação conjunta de profissionais, em que os enfermeiros realizam um papel fundamental devido a sua proximidade com a paciente (SANTOS FK, et al., 2020). Trata-se de promover uma ação de rastreamento e monitoramento dessa patologia durante todas as fases, especialmente no puerpério. 
Analisando-se os artigos científicos, verifica-se que essa temática não é objeto de atenção no sistema de saúde públicos e privados, em que o tratamento especializado à puérpera acometida pela DPP encontra-se inexistente, apresentando cuidados fragmentados e sem padronização resolutiva (OLIVEIRA AM, et al., 2016). Portanto, as instituições de saúde desconhecem estratégias efetivas de enfrentamento preventivo à depressão puerperal. Conforme o autor supracitado, a medicalização é o principal método de tratamento oferecido às mulheres em seu estágio mais evoluído da doença. Assim, as condições de acolhimento à mulher em seu estado gravídico-puerperal apresentam-se insuficientes para atender a demanda de assistência preventiva, carecendo de planos integrados de cuidado efetivo à parturiente.

Além dos riscos inerentes à realidade pessoal da parturiente, quando a DPP não é diagnosticada aumentase a probabilidade de outros riscos à gestação e no pós-parto, especialmente relacionado ao uso de substâncias nocivas ao seu organismo, desnutrição, dificuldade para seguir as instruções médicas, diminuição da regularidade de consultas. (AYOAMA EA, et al., 2019). Ainda, isso reflete diretamente na gestação, aumentando o risco de mortalidade dos neonatos. Assim, a mulher acometida por essa patologia não se encontra em condições psíquicas para perceber os fatos relevantes ao seu problema, sendo necessário um profissional externo. Portanto, a atuação do profissional de enfermagem é fundamental nesse processo de identificação da DPP, pois desde as primeiras consultas nas unidades de saúde, é possível iniciar procedimentos investigativos relativos aos fatores de riscos.

Nesse sentido, verificou-se na pesquisa que é necessário o estabelecimento de uma equipe multidisciplinar especializada a fim de auxiliar a gestante em todas as fases do ciclo gravídico-puerperal, diligenciando-se pela investigação dos fatores de risco associados à DPP (ALMEIDA NMC e ARRAIS AR, 2016). Busca-se rastrear esses fatores na relação entre mãe e bebê, bem como criar um ambiente de maior confiança entre os profissionais da saúde envolvido no processo de acolhimento. Conforme os autores supracitados, a presença de um psicólogo é essencial para a intermediação efetiva entre a paciente e os profissionais de enfermagem. Esse atendimento multidisciplinar é necessário em razão da complexidade da relação entre a gestante, seu bebê e os demais profissionais da saúde.

Conforme a pesquisa realizada nas produções científicas, a depressão puerperal é uma patologia que pode apresentar indícios desde o início da gestação, sendo fundamental a sua atenção desde o pré-natal (GONÇALVES FBAC e ALMEIDA MC, 2019). Portanto, é indispensável a realização de uma assistência de qualidade, bem como orientações a fim de prevenir o surgimento ou agravamento da DPP. Conforme constado pelos autores supracitados, essa doença pode apresentar-se em diversos níveis de intensidade, exigindo dos profissionais de enfermagem maior sensibilidade no processo de identificação e orientação à paciente. São diversas variáveis que dificultam o tratamento preventivo desse transtorno, principalmente a falta de capacitação especializada ofertada aos profissionais de enfermagem e sua respectiva estruturação (TOLENTINO E, et al., 2016).

Os aspectos subjetivos e psicológicos que envolvem o ciclo gravídico-puerperal são complexos, o que implica em uma busca "não uniforme" para atender às necessidades da puérpera (RIBEIRO NM, et al., 2019). Nesse contexto, todos os atores envolvidos nesse processo são afetados direta ou indiretamente pelas variantes emocionais e psicológicas da mulher. Conforme o autor supracitado, o nascimento de um bebê é um acontecimento de natureza social, gerando nos mais próximos diversas expectativas em relação a esse fato. Consequentemente, os conflitos internos e externos tendem a aumentar em cada núcleo familiar, conforma as suas próprias peculiaridades.

Os profissionais de enfermagem são aliados altamente relevantes nesse momento, atuando não apenas como profissionais da saúde em seu sentido biológico, mas também sob seu aspecto psíquico e mental (TOLENTINO E, et al., 2016). Portanto, não possuindo o objetivo de substituir o profissional da psicologia, a assistência de enfermagem pode proporcionar à puérpera o apoio preliminar em relação as suas necessidades imediatas. Pois, diante da imprevisibilidade do surgimento da depressão puerperal, a qualidade no acolhimento da mulher em suas diversas fases do ciclo gravídico-puerperal é essencial para a consecução de seu objetivo (GONÇALVES FBAC e ALMEIDA MC, 2019).

Mesmo diante dos esforços realizados pelos profissionais de enfermagem e sua atenção ao acolhimento humanizado dessas mulheres, muitos elementos demográficos, sociais e econômicos influenciam no 
surgimento ou agravamento da DPP (BOSKA GA, et al., 2016). Desse modo, conforme o autor supracitado, a patologia predomina em jovens de 20 a 24 anos de idade, casas e com baixo grau de escolaridade. Assim, a cultura na qual encontra-se inserida a puérpera é essencial para determinar suas condições psicológicas no pós-parto, representando um marco em sua vida.

Consequentemente, a saúde mental da mulher consiste em uma das principais preocupações em seu ciclo gravídico-puerperal, a qual é habitualmente negligenciada pelos profissionais da saúde (JORDÃO RRR, et al., 2017). Conduto, é essencial que a puérpera receba assistência em todas as fases, buscando a atuação de forma preventiva e efetiva (MOLL MF, et al., 2019). Assim, é importante a atenção de todos os aspectos que envolvem a depressão puerperal, a fim de evitar o seu tratamento tão somente nas fases mais graves e avançadas da patologia.

\section{CONSIDERAÇÕES FINAIS}

Em análise das produções científicas coletadas e selecionadas, verificou-se que a maioria dos autores consideram que a atuação do profissional de enfermagem é fundamental para a prevenção da DPP, especialmente em relação aos fatores de risco de sua realidade. Os principais resultados na pesquisa realizada demonstram que não existe uma infraestrutura adequada para a atuação do profissional de enfermagem no Brasil para acolher de forma preventiva a paciente mediante a identificação e o agravamento da Depressão Puerperal. A efetiva prevenção às gestantes e puérperas depende diretamente desses profissionais, os quais podem realizar o acolhimento humanizado, instruindo acerca da patologia e encaminhá-las para setores específicos para atender a sua necessidade.

\section{REFERÊNCIAS}

1. ALMEIDA NMC, ARRAIS AR. O Pré-Natal Psicológico como Programa de Prevenção à Depressão Pós-Parto. Psicologia: Ciência e Profissão, 2016; 36(4): 847-863.

2. ALOISE SR, et al. Depressão pós-parto: identificação de sinais, sintomas e fatores associados em maternidade de referência em Manaus. Enfermagem em Foco, 2019; 10(3): 41-45.

3. AYOAMA EA, et al. A importância do profissional de enfermagem qualificado para detecção da depressão gestacional. Braz. J. Hea. Rev., 2019; 2(1): 177-184.

4. BOSKA GA, et al. Sintomas depressivos no período puerperal: identificação pela escala de depressão pós-parto de Edinburgh. J Nurs Health, 2016; 1(1): 38-50.

5. FIOCRUZ. Fundação Oswaldo Fruz. Depressão pós-parto acomete mais de 25\% das mães no Brasil. 2016. Disponível em: <https://portal.fiocruz.br/noticia/depressao-pos-parto-acomete-mais-de-25-das-maes-no-brasil>. Acesso em: 2 de março de 2021.

6. GOMES BKA, et al. Assistência de enfermagem na prevenção e atenção à mulher com depressão pós-parto. Ciências Biológicas e de Saúde Unit, 2019; 5(2): 121-136.

7. GONÇALVES FBAC, ALMEIDA MC. A Atuação da Enfermagem Frente à Prevenção da Depressão Pós-Parto. Ensaios e Ciênc., 2019; 23(2): 140-147.

8. GUIMARÃES RB, et al. Atuação do enfermeiro à gestante e puérpera com depressão. Revista Eletrônica Acervo Saúde, 2021; 13(1): e5178.

9. JORDẤ RRR, et al. Acurácia das características definidoras do diagnóstico de enfermagem Desempenho do Papel Ineficaz. Rev. Eletr. Enf., 2017; 19(1): a10.

10. MENDES KDS, et al. Revisão integrativa: método de pesquisa para a incorporação de evidências na saúde e na enfermagem. Texto contexto - enferm., 2008; 17(4): 758-764.

11. MOLL MF, et al. Rastreando a depressão pós-parto em mulheres jovens. Rev. Enferm. UFPE On Line, 2019; 13(5):1338-1344.

12. OLIVEIRA AM, et al. Conhecimento de profissionais da Estratégia Saúde da Família sobre depressão pós-parto. J Nurs Health, 2016; 1(1):17-26.

13. RIBEIRO NM, et al. Assistência de enfermagem na depressão pós-parto. Revista Científica Interdisciplinar, 2019; 4(1):a9.

14. SANTOS FK, et al. Percepção de enfermeiros sobre diagnóstico e acompanhamento de mulheres com depressão pós-parto. Revista Nursing, 2020; 23(271): 4999-5005.

15. SILVA CRA, et al. Depressão pós-parto: a importância da detecção precoce e intervenções de enfermagem. ReBIS [Internet], 2020; 2(2):12-19.

16. SOUZA KLC, et al. Conhecimento de enfermeiros da atenção básica acerca da depressão puerperal. Rev. Enferm. UFPE Online, 2018; 12(11): 2933-2943.

17. SOUZA MT, et al. Revisão integrativa: o que é e como fazer. Einstein, 2010; 8(1):102-106.

18. TOLENTINO E, et al. Depressão pós-parto: conhecimento sobre os sinais e sintomas em puérperas. Revista de Ciências da Saúde Nova Esperança, 2016; 14(1): 59-66.

19. VIANA, MDZS, et al. Estratégias de enfermagem na prevenção da depressão pós-parto. R. pesq.: cuid. fundam. Online, 2020; 12: 953-957. 